SCIENTIFIC LETTER

\title{
Impact of first line radiofrequency ablation in patients with lone atrial flutter on the long term risk of subsequent atrial fibrillation
}

\author{
A Da Costa, C Romeyer-Bouchard, N Zarqane-Sliman, M Messier, B Samuel, A Kihel, E Faure, \\ K Isaaz
}

$\mathrm{R}$ adiofrequency catheter ablation (RFA) of the inferior vena cava-tricuspid isthmus is the treatment of choice for atrial flutter (AFL) given its high efficacy. One controlled study of AFL with medication has been done to date. ${ }^{1}$ Despite the introduction and high success rate of periablation, questions remained: is transisthmus ablation a definite cure for patients with AFL or can their follow up be characterised by the occurrence of other atrial arrhythmia? Many studies, some recent, have tried to answer these questions $^{23}$ but one question remains: will first line transisthmus ablation alter the long term risk of atrial fibrillation $(\mathrm{AF})$ ? The aim of this study was to evaluate the long term risk of subsequent AF after RFA in two groups of patients $<80$ years old with lone AFL: group 1, receiving first line RFA treatment; and group 2, receiving RFA after failure of at least one drug treatment after restoration of sinus rhythm. By convention, lone AFL excluded prior evidence of AF.

\section{PATIENTS AND METHODS}

Over 44 months, 318 patients were referred for RFA treatment for AFL (February 1998 to October 2001). Only 176 patients $(55.3 \%)$ were enrolled. Inclusion criteria were as follows: typical AFL on the ECG; typical intracardiac electrogram activation sequence reported by analysis of the activation sequence with a dodecapolar catheter; evidence of isthmus participation during entrainment manoeuvres; and prior evidence of AF excluded by lone AFL. Exclusion criteria were a documented history of $\mathrm{AF}$ and age $\geqslant 80$ years old. Patients were enrolled in group 1 if the first episode of AFL was treated by RFA (first line treatment). Patients were enrolled in group 2 when RFA was applied after at least one documented recurrence after medication following sinus

rhythm restoration. Secondary prevention relied on drug treatment. Of the 176 patients aged 63 (11) years (33 women) with lone AFL, 93 patients were in group 1 (64 (11) years) and 83 were in group 2 (61 (10) years). Of the initial 318 referred patients, $142(44.7 \%)$ were excluded because of either age ( 25 patients) or a history of AF (117 patients). The procedural end point was defined as a complete bidirectional isthmus block. ${ }^{4}$ Cumulative risk of AF was determined by outpatient follow up, on recurring symptoms or palpitations, and at the end of the study (December 2002). The mean follow up was evaluated by the log rank test and KaplanMeier method. The data are presented as mean (SD).

\section{RESULTS}

Clinical presentation and electrophysiological data were as follows: age (63 (11) years); sex (18.75\% women); AFL duration since onset (13 (23) months), structural heart disease $(45.5 \%)$, left ventricular ejection fraction (58.6 $(12) \%)$, and left atrial size (42 (7) $\mathrm{mm})$. The mean duration of radiofrequency applications (minutes) was 14.6 (17) (range 1.5-120). A bidirectional isthmus conduction block was obtained in 171 of 176 patients $(97.2 \%)$ and an incomplete block in five patients $(2.8 \%)$. One local complication due to an accidental arterial puncture was noted (groin haematoma requiring surgical treatment; group 2). An AFL recurrence was documented in 11 of $170(6.5 \%)$ patients. Of the 176 consecutive patients undergoing RFA for AFL, long term follow up was obtained for 170 (96.6\%). Six patients were lost to follow up, three in group 1 and three in group 2 (not significant). During the outcome, 33 atrial arrhythmic

Abbreviations: $A F$, atrial fibrillation; $A F L$, atrial flutter; RFA, radiofrequency catheter ablation

Table 1 Comparison between group 1 and group 2

\begin{tabular}{llll}
\hline & Group 1 (n=93) & Group 2 (n=83) & p Value \\
\hline Age (years) & $64(11)$ & $61(10)$ & NS \\
Structural heart disease & $49.5 \%$ & $41 \%$ & NS \\
Left ventricular ejection fraction (\%) & $57(12)$ & $60(11)$ & NS \\
Left atrial size (mm) & $43(7)$ & $40(6)$ & NS \\
AFL duration since onset (months) & $1.6(1.4)$ & $27(29)$ & $<0.0001$ \\
Bidirectional block & $97.8 \%$ & $96.25 \%$ & $\mathrm{NS}$ \\
Duration of RFA application (minutes) & $13.7(14)$ & $15.8(19)$ & $\mathrm{NS}$ \\
Antiarrhythmic agents at end of follow up & $23 / 90$ & $23 / 80$ & $\mathrm{NS}$ \\
Mean follow up (months) & $22(12)$ & $25(15)$ & 0.1 \\
Overall atrial arrhythmias & $24.4 \%(22 / 90)$ & $27.5 \%(22 / 80)$ & $\mathrm{NS}$ \\
AFL recurrence & $6.7 \%(6 / 90)$ & $6.25 \%(5 / 80)$ & $\mathrm{NS}$ \\
Atrial tachycardia occurrence & $4.4 \%(4 / 90)$ & $3.75 \%(3 / 80)$ & $\mathrm{NS}$ \\
Atrial fibrillation occurrence & $13.3 \%(12 / 90)$ & $17.5 \%(14 / 80)$ & 0.9 \\
Complication & 0 & 1 & $\mathrm{NA}$ \\
\hline
\end{tabular}

AFL, atrial flutter; NA, not applicable; NS, not significant; RFA, radiofrequency catheter ablation. 
events occurred: AF in 26 of 170 patients (15.3\%) and atrial tachycardia in seven of 170 patients $(4.1 \%)$. Table 1 compares characteristics and treatment of group 1 and group 2 .

\section{DISCUSSION}

The risk of AF following RFA for typical AFL has been appreciated for some time. The risk factors for recurrence have been identified as atypical flutter or a history of $\mathrm{AF}$ before ablation, abnormal left ventricular ejection fraction, and inducible $\mathrm{AF}^{2}{ }^{35}$ Without these factors, the risk of $\mathrm{AF}$ is believed to be relatively low. The question remained whether first line transisthmic ablation would alter the long term risk of AF. Our study addressed this question and showed that despite a high periablation success rate of transisthmic catheter ablation, first line RFA of lone AFL does not alter the long term risk of subsequent AF. Some controversies exist regarding the risk of $\mathrm{AF}$ in patients without a history of $\mathrm{AF}$ (lone AFL). Results underlined a low risk with a prevalence close to $10 \%$ after mid term follow up. ${ }^{23}$ In a report from the Cleveland Clinic, however, the long term risk of AF after cavo-tricuspid isthmus RFA of AFL in patients with no history of AF was actually found to be quite high (77\%). ${ }^{2}$ The mean follow up in that study was 36 (15) months, longer than in any previously reported studies. ${ }^{2}$ A retrospective study concerning medically treated AFL determined a $48 \%$ risk of AF within 10 years. ${ }^{5}$ That study found several variables associated with AF risk: sex (women being at a higher risk), age, hypertension, diabetes, and recurrent AFL. Only diabetes and recurrent AFL were independent predictors of AF. ${ }^{5}$ To that point, it was unknown whether first line RFA altered the long term risk of AF. Our study determined that first line RFA treatment of lone AFL does not reduce the long term risk of subsequent AF in patients with lone AFL. The prevalence of AF after ablated AFL in patients with lone AFL was found to be close to $15 \%$ after two years.

\section{Authors' affiliations}

A Da Costa, C Romeyer-Bouchard, N Zarqane-Sliman, M Messier, B Samuel, A Kihel, E Faure, K Isaaz, Division of Cardiology, University Jean Monnet, Saint-Etienne, France

Presented in part at the Annual Scientific Sessions of the American Heart Association; 17-20 November 2002, Chicago, Illinois, USA.

Correspondence to: Dr Da Costa, Service de Cardiologie, Hôpital Nord, Centre Hospitalier Universitaire de Saint-Etienne, 42055 SaintEtienne Cedex 2, France; dakosta@aol.com

Accepted 2 April 2004

\section{REFERENCES}

1 Natale A, Newby KH, Pisano E, et al. Prospective randomized comparison of antiarrhythmic therapy versus first line radiofrequency ablation in patients with atrial flutter. J Am Coll Cardiol 2000;35:1898-904.

2 Gilligan DM, Zakaib JS, Fuller I, et al. Long term outcome of patients after successful radiofrequency ablation for typical atrial flutter. Pacing Clin Electrophysiol 2003;26:53-8.

3 Paydak H, Kall JG, Burke MC, et al. Atrial fibrillation after radiofrequency ablation of type I atrial flutter: time to onset, determinants, and clinical course. Circulation 1998:98:315-22.

4 Chen J, De Chillou C, Basiouny T, et al. Cavotricuspid isthmus mapping to assess bidirectional block during common atrial flutter radiofrequency ablation. Circulation 1999;100:2507-13.

5 Halligan S, Maurer M, Munger T, et al. Risk and predictors of subsequent atrial fibrillation in patients presenting with typical atrial flutter [abstract]. Circulation 2001;104:11714.

\section{IMAGES IN CARDIOLOGY}

Immediate endovascular stent graft repair of acute thoracic aortic rupture caused by blunt trauma

W e present a case of trauma induced thoracic aortic rupture treated by immediate graft stenting. A 22 year old male victim of a head-on collision between his motorcycle and a car presented to the trauma unit. Chest $x$ ray showed widening of the mediastinum, and the patient was referred for total body computed tomography. Findings revealed a traumatic aortic tear (panel A, arrow), and injuries to the head, kidney, and pelvis. Further evaluation by aortic injection in the invasive radiology unit confirmed the diagnosis of traumatic aortic tear with pseudoaneurysm formation (panel B, arrow).

Treatment consisted of a $24 \mathrm{~mm}$ covered stent graft, introduced through the femoral artery and placed at the tear site (panel C).

Repeated aortic injection after the procedure demonstrated no leakage of contrast material outside the aorta. The patient remained haemodynamically stable and slowly recovered from his additional injuries.
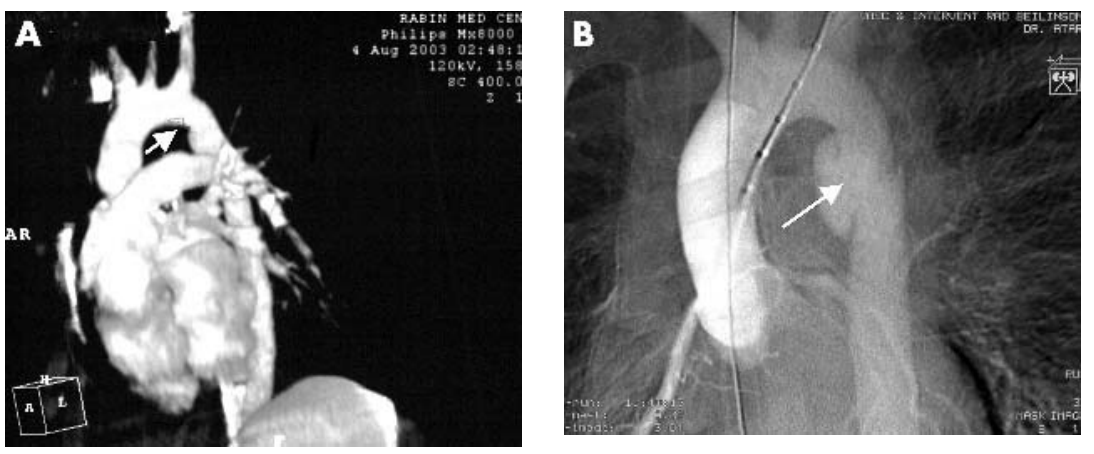

On follow up transoesophageal echocardiography, the stent appeared in place with no enlargement or leakage of the pseudoaneurysm.

We feel that this technique should be considered as an alternative to open surgery in selected cases.
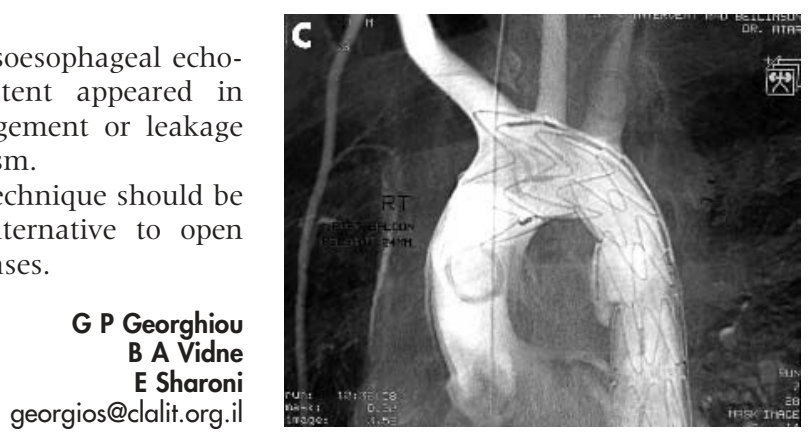